\title{
Saponosides stéroïdiques de l'aubergine (Solanum melongena L.) I. Intérêt alimentaire, méthodologie d'analyse, localisation dans le fruit
}

\author{
S. Aubert ${ }^{1}$, M. C. Daunay ${ }^{2}$ et E. Pochard ${ }^{2}$ \\ INRA, centre de recherches d'Avignon, Station de technologie des produits végétaux, Domaine Saint-Paul, B.P. 91 F84140 \\ Montfavet; \\ INRA, centre de recherches d'Avignon, Station d'amélioration des plantes maraîchères, Domaine Saint-Paul, B.P. 91, F84140 \\ Montiavet, France.
}

(reçu le 27 juillet 1987, accepté le 20 avril 1989)

Résumé - Le fruit de l'aubergine possède une saveur caractéristique pouvant déplaire aux consommateurs. Des risques, mais aussi des avantages, pour la qualité alimentaire de ce légume, sont liés à la présence dans la pulpe, de deux types de saponosides à noyau stéroïdique: d'une part, des glycoalcaloïdes du type solasonine, dans la zone placentaire, et, d'autre part, des composés saponosides sans noyau hétérocyclique azoté, localisés plus particulièrement dans les graines, mais trouvés aussi dans la zone de pulpe sans graines apparentes.

Une technique usuelle d'extraction-purification est proposée à l'usage des sélectionneurs. Les teneurs en ces substances sont évaluées par une méthode colorimétrique classique (réaction de Clarke) et après leur révélation spécifique en chromatographie sur couche mince dans une analyse semi-quantitative. Les activités hémolytiques et tensioactives des extraits obtenus sur diverses variétés, lignées et hybrides, sont vérifiées.

La relation entre la teneur en ces substances et la sapidité amère, typique de ce légume-fruit est mise en évidence. Les concentrations en glycoalcaloïdes, sans tenir compte des saponosides non azotés, sont voisines de celles de la solanine dans les tubercules de pommes de terre. Les limites d'acceptabilité gustative, l'intérêt diététique et culinaire de ces substances sont discutés.

solanacées - saponines - alcaloïdes - qualité organoleptique

Summary - Saponins and steroidic alcaloids of Egg-plant (Solanum melongena L.) I. Food interest, analytical methodology, localisation in fruit. Egg-plant fruit contains a characteristic flavor which can be disliked by some consumers. Risks, but also advantages, in food quality of this vegetable are linked to two classes of compounds with saponin steroidic structure. The first one, glycoalkaloids with solasonin structure, characteristic of this solanaceous plant, are concentrated near placentar pulp. The second, contains saponins without a nitrogen heterocyclic structure, compounds localised in the seeds are revealed also in the pulp without perceptible seeds.

Usual extraction and purification procedure is proposed for breeding use. Contents of these substances are evaluated by a classical colorimetric method (Clarke's reaction) and after specific revelation on thin layer chromatography in relative quantitative analysis. Hemolytic activity and foaming properties of extracts are controled for a few varieties, lines and hybrids.

Relationships between the content of these substances and the typical bitterness of these fruits are investigated. With the exception at the saponins which have a oxygenated heterocyclic structure, the glycoalkaloids are close to the structure of the potato solanins. Organoleptic acceptability threshold, dietetic and culinary aspects of these substances are discussed. 


\section{INTRODUCTION}

Le nom italien de l'aubergine, melanzana ou mela insana, traduit par "pomme malsaine", symbolise bien la méfiance des consommateurs pour cette solanacée. Quelques vertus médicinales lui sont aussi attribuées (Arveiller, 1970). De nos jours, les opinions divergent encore sur les qualités sanitaires de ce légume-fruit : effets toxiques attribués aux alcaloïdes ((Claringbold et al., 1982; Dalvie \& Bovie, 1984; Kibler et al., 1985) ou vertus pharmacologiques et diététiques (Mitschek, 1970; Kritchevski et al., 1975; Vohora et al., 1984). La composition biochimique et la valeur alimentaire du fruit de l'aubergine (Solanum melongena L.) sont encore mal connues. En particulier, c'est le cas pour les facteurs responsables de sa saveur singulière: sapidité forte, typique, à dominante amère (Aubert, 1971; Aubert \& Pochard, 1981; Jones \& Fenwick, 1981), qui rebute parfois les consommateurs.

L'objectif de cette étude est d'analyser les saponosides stéroïdiques de l'aubergine (alcaloïdes et saponosides non azotés), qui sont des constituants primordiaux de la qualité alimentaire de ce légume. Nous rechercherons dans quelle mesure les différences variétales de saveur sont liées aux teneurs en ces substances.

Dans une première étape, les difficultés méthodologiques d'une telle analyse seront explicitées. Elles sont, en partie, dues aux faibles concentrations de ces substances dans les fruits commercialisables, car les lots cueillis trop tardivement sont rejetés par les consommateurs.

Les limites d'acceptabilité ne reposent pas sur des critères objectifs et usuels pour les sélectionneurs. Sur quelques variétés typiques, nous étudierons les différences de concentration selon les zones de pulpe prélevée, plus particulièrement dans le mésocarpe renfermant plus ou moins de graines apparentes, et suivant la maturité du fruit.

Dans une deuxième partie de cette étude, nous étudierons d'autres sources de variation de la teneur des fruits en saponosides stéroïdiques, en particulier les différences liées au génotype et les fluctuations dues aux conditions agroclimatiques de culture et de récolte.

\section{Mise au point bibliographique}

\section{Nature des saponosides de l'aubergine}

On trouve dans le fruit de l'aubergine deux types de saponosides stéroïdiques ("saponines" en anglais): des saponosides azotés (glycoalca- loïdes) et des saponosides non azotés. La dénomination "glucoalcaloïde" employée dans les publications françaises peut prêter à confusion sur la nature des hétérosides liés. Nous préférons utiliser le terme "glycoalcaloïde» (GA), plus général.

La structure moléculaire des glycoalcaloïdes (GA) est caractérisée par un noyau stéroïdique porteur d'un hétérocycle azoté, ou aglycone (synonymes: alcamine ou génine), auquel est accroché un radical hétéroside. Les aglycones, présentant diverses formes, ou les radicaux hétérosides, composés de diverses associations de sucres, sont spécifiques de chaque espèce de Solanacées (Schreiber, 1968) (Fig. 1a).

De Wall et al., (1960) ont identifié l'aglycone solasodine dans le fruit de l'aubergine. Cet alcaloïde est quelquefois encore confondu dans la littérature avec la solanidine de la pomme de terre ou la tomatidine de la tomate. Car, les glycoalcaloïdes, solanine et tomatine, sont aussi impliqués dans les problèmes d'amertume et de toxicité de ces deux espèces de la même famille (Maga, 1980; Aubert, 1981).

Les saponosides stéroïdiques non azotés (SNA) ont une structure moléculaire très proche de celle des glycoalcaloïdes, mais leur aglycone est dépourvu d'hétérocycle azoté (fig. 1b).

Récemment, Kintia \& Shvets (1985 a \& b), sur des extraits de graines d'aubergine, ont détecté la présence de plusieurs saponosides non azotés (melongosides), dont les structures sont : soit du type spirostanol (melongosides $L$ et $M$ ), soit du type furostanol (melongosides $\mathrm{N}, \mathrm{O}$ et $\mathrm{P}$ ). Leurs aglycones sont : soit la tigogénine, soit la diosgénine. Comme les alcaloïdes, ils présentent un intérêt pour l'industrie pharmaceutique, car ils servent à l'hémisynthèse de composés ayant un noyau stéroïdique et doués d'activité hormonale (Baccou et al., 1983; Regerat \& Pourrat, 1981; Trione \& Cony, 1988).

Du fait de leurs structures moléculaires voisines, les saponosides non azotés et les glycoalcaloïdes ont des caractéristiques communes: amertume, propriétés tensioactives, effets physiologiques sur les êtres vivants (Birk \& Peri, 1980; Oakenfull, 1981). Par exemple, notons certains effets intéressants sur le plan médical: abaissement du taux de cholestérol plasmatique et élimination des acides biliaires dans le transit intestinal (Oakenfull \& Sidhu, 1983; Sidhu \& Oakenfull, 1986), avec modification des activités cholinestérase et lipase (Mahato et al., 1982).

Dans le domaine de la nutrition animale, ces saponosides stéroïdiques interviennent aussi dans l'appétence pour les végétaux qui les contiennent et peuvent avoir un effet antinutri- 


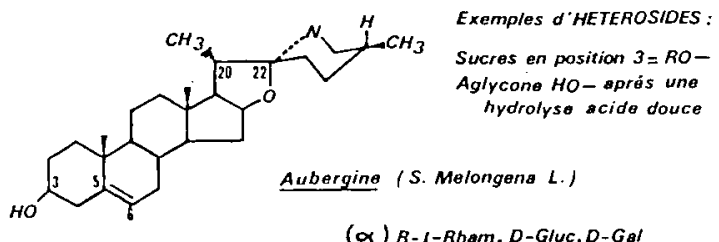

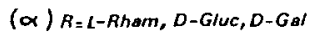
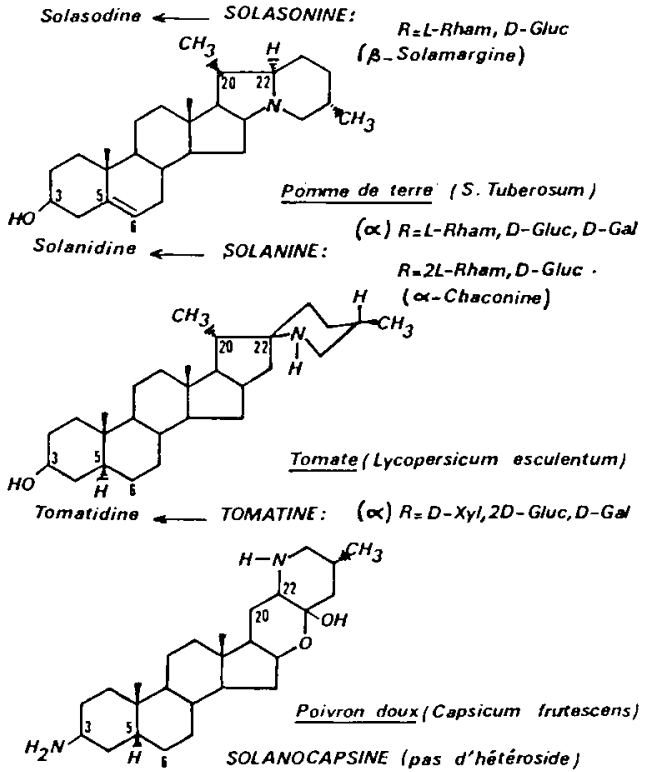

Fig. 1a. Alcaloïdes stéroïdiques de Solanacées (Schreiber, 1968).

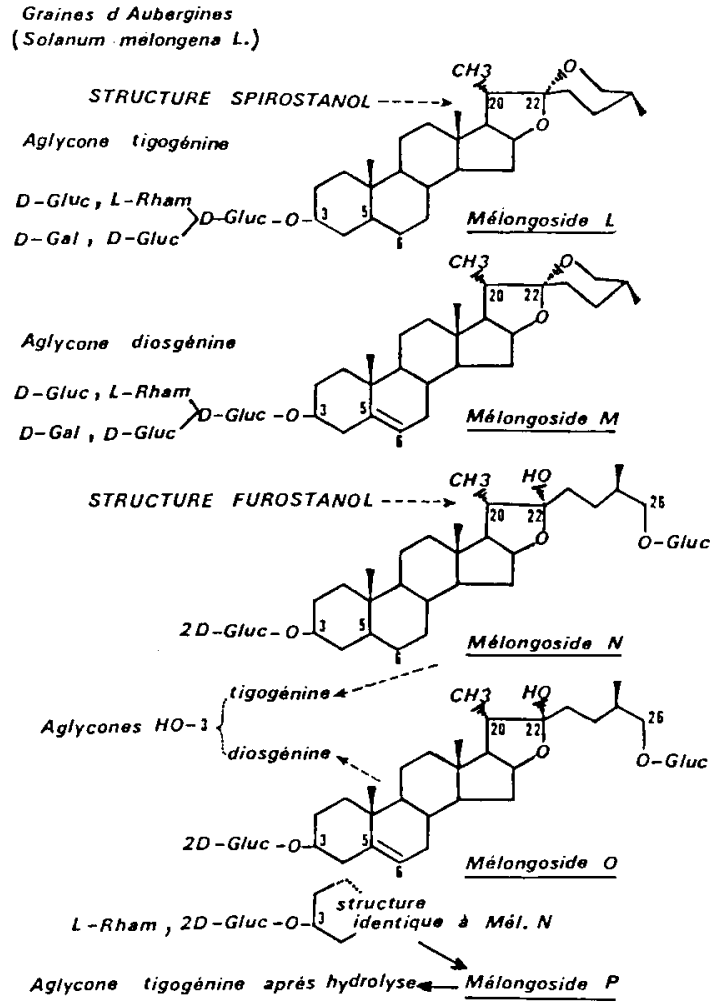

Fig. 1b. Saponosides stéroïdiques non azotés (Kintia \& Shvets, 1985).

Fig. 1a et 1b. Quelques structures chimiques de saponines stéroïdiques de Solanacées.

tionnel (Cheeke, 1976; Freeland et al., 1985; Morgan et al., 1972).

\section{Méthodologie d'extraction et d'analyse}

Les méthodes d'étude des glycoalcaloïdes font l'objet d'une abondante littérature déjà ancienne (Schreiber, 1968). En France, Verbist et al., 1977; Verbist \& Monnet (1979) ont inventorié et discuté de façon critique les différentes techniques d'extraction, de fractionnement et de dosage des glycoalcaloïdes de diverses solanacées. Crabbe \& Fryer $(1980,1982)$ ont passé en revue les principales difficultés d'extraction et de dosage des dérivés de la solasodine, substance qui nous intéresse plus particulièrement, puisque c'est l'alcaloïde propre à l'aubergine. Les méthodes d'analyse des saponosides non azotés sont beaucoup plus rarement décrites (Fenwick \& Oakenfull, 1983; Price et al., 1987). L'étude de la séquence des sucres fixés à l'aglycone présente aussi quelques difficultés (Massiot et al., 1986).

La chromatographie liquide à haute performance, ainsi que celle en phase gazeuse à haute résolution, sont des voies d'analyse classiques, néanmoins discutables dans notre contexte de travail. En effet, ces techniques, pour fournir des dosages précis, nécessitent des purifications poussées, et exigent la maîtrise, de façon reproductible, des conditions d'hydrolyse des hétérosides (Gelder, 1984). La détection est peu spécifique dans le court ultraviolet, tout comme celle des hétérosides en réfractométrie. De même, la radio-immunochimie (Weiler et al., 1980), intéressante du fait de sa bonne spécificité du dosage de la solasodine, est de mise en œuvre relativement délicate.

\section{Objectifs et choix analytiques}

Nous avons recherché des techniques usuelles d'analyse des saponosides stéroïdiques de l'aubergine, à la portée de laboratoires sommairement équipés : colorimétrie, activité hémolytique, indice de mousse et chromatographie sur couche mince (C.C.M). Avec la même préoccupation, Douillard \& Marion (1987) ont proposé une technique usuelle de mesure du pouvoir hémolytique, à l'usage des sélectionneurs de luzerne et des spécialistes de l'alimentation animale.

Deux voies d'analyses de routine ont été adoptées. D'une part, la CCM permet diverses adaptations grâce à quelques révélateurs relativement spécifiques de chaque type de saponoside, notamment sur les différences de structure 
de l'aglycone. La réactivité porte généralement sur la fonction alcaloïde, sur les noyaux stéroïdiques et sur les heitérosides (Price et al., 1987; Wagner et al., 1984). D'autre part, on a choisi une technique colorimétrique très classique (Wu \& Salunkhe, 1976) de dosage des glycoalcaloïdes, du type solanine ou solasonine présentant une double liaison 5-6 sur le cycle B du noyau stéroïde (fig. 1), avec quelques adaptations précisées ultérieurement. Cette méthode, utilisant le réactif de Clarke, à l'avantage d'être bien connue, et est couramment utilisée par les sélectionneurs sur la pomme de terre ou pour l'industrie de ses produits de transformation.

\section{MATÉRIEL ET MÉTHODES}

\section{Matériel végétal et échantillonnage}

Trois variétés ont été choisies, en raison de leurs caractères organoleptiques très contrastés (Aubert, 1971) :

- «Violette de Barbentane" et "Ronde de Valence», à la peau noir violacé et à la chair verte; ces variétés ont une sapidité relativement forte, avant comme après cuisson, surtout la seconde.

- «Dourga», à la peau et à la chair blanches, est une variété de saveur particulièrement douce, presque totalement dépourvue d'amertume.

En outre, ont été étudiées deux autres variétés à fruits blancs, «C8» et «H5», ainsi que les hybrides $\mathrm{F} 1$ réciproques entre "Ronde de Valence" et “Dourga": «F1: Dourga X Ronde de Valence» et «F1: Ronde de Valence X Dourga».

Les fruits proviennent de divers essais conduits à la station d'amélioration des plantes maraîchères de l'INRA du Centre d'Avignon-Montfavet, en serre de printemps, ou en culture estivale de plein champ irriguée.

L'échantillonnage pour une analyse est compris entre 5 et 8 fruits, selon leur grosseur. Une attention particulière est portée à l'homogénéité de stade physiologique des fruits d'un même échantillonnage : fruits "jeunes" (stade commercialisable) ou fruits "vieux" (ayant dépassé ce stade). Les fruits "vieux" se distinguent aisément des fruits “jeunes»: par la couleur terne de leur épiderme (violet clair au lieu du noir intense habituel), par une texture particulièrement ferme au niveau du pédoncule et par la présence de graines brunes et dures, désagréables pour le consommateur.

Les prélèvements de chair sont effectués sur des fruits venant d'être cueillis ou ayant été conservés quelques jours à $8-10^{\circ} \mathrm{C}$ et à plus de $80 \%$ d'humidité relative.

Trois zones du fruit sont prélevées :

- l'épicarpe (zone épidermique, généralement éliminée),

- le mésocarpe avec graines (pulpe placentaire),

- le mésocarpe sans graines (pulpe située dans le tiers inférieur du fruit, vers le pédoncule).

\section{Protocole général d'analyse}

Nous avons utilisé la méthodologie classique d'Oakenfull (1981) et Price et al. (1987), avec quelques adaptations techniques précisées ici. Les glycoalcaloïdes (GA) et les saponosides non azotés (SNA) sont extraits, puis purifiés ensemble. Leurs teneurs respectives sont ensuite évaluées par colorimétrie ou par chromatographie sur couche mince (CCM).

\section{Extraction des saponosides stéroïdiques totaux}

Les fruits sont découpés en onglets longitudinaux dont on élimine l'épiderme coloré, de manière à prélever environ $250 \mathrm{~g}$ de matière fraîche par échantillon.

L'extraction s'effectue par broyage fin dans un "mixer" (Vorwerk, $18000 \mathrm{t} / \mathrm{min}$ pendant une min), d'une partie de matière fraîche pour quatre parties d'éthanol $\left(96^{\circ}\right)$. La désintégration des téguments bruns des graines dures (cas des fruits "vieux") nécessite parfois un broyage plus poussé (Omni-Mixer Sorvall). Afin d'améliorer la diffusion, le mélange pulpeux est agité pendant 15 à $16 \mathrm{~h}$ au froid $\left(4\right.$ à $\left.10^{\circ} \mathrm{C}\right)$. On filtre ensuite, sur papier (éventuellement sur lit de célite si nécessaire) jusqu'à obtention d'un liquide limpide. L'alcool est chassé sous vide à température inférieure à $45^{\circ} \mathrm{C}$. Le résidu aqueux (environ $30 \mathrm{ml}$ ) est amené à $\mathrm{pH} 3$ par apport d'acide chlorhydrique dilué, afin d'assurer une bonne solubilisation des saponosides basiques.

A ce stade, on peut décolorer et purifier l'extrait aqueux par quelques lavages à l'éther de pétrole, au chloroforme ou à l'acétate d'éthyle. Mais cette opération peut occasionner une perte en aglycones. Elle n'est à conseiller que dans un but de meilleure séparation des hétérosides.

On filtre ensuite sous vide sur lit de célite (type 545 Prolabo) et on ajuste le filtrat à $50 \mathrm{ml}$ avec de l'eau. Sur une fraction de $25 \mathrm{ml}$ (double dosage éventuel), on ramène le $\mathrm{pH}$ à 9 avec 1 à $2 \mathrm{ml}$ d'une solution concentrée de soude; puis on ajoute $10 \mathrm{ml}$ d'alcool isobutylique (butanol secondaire rectapur Prolabo) en agitant 1 à 2 min. Après $2 \mathrm{~h}$ de décantation, entre 4 à $10^{\circ} \mathrm{C}$, on obtient une phase aqueuse brune et un surnageant isobutanolique jaune-vert clair limpide. En tenant compte de la fraction du solvant miscible dans l'eau, le volume d'extrait final considéré est de $10 \mathrm{ml}$.

\section{Dosage colorimétrique des glycoalcaloïdes (GA)}

On prélève de 0,2 à $2 \mathrm{ml}$ de l'extrait isobutanolique décanté. L'alcool est évaporé, en tube rodé, à température inférieur à $40^{\circ} \mathrm{C}$, sous vide. Puis, on ajoute en agitant, $10 \mathrm{ml}$ de réactif de Clarke (formol officinal 0,2 $\mathrm{ml}$ pour $100 \mathrm{ml}$ d'acide orthophosphorique). Après activation à la lumière et repos $1 \mathrm{~h}$ pour clarification du milieu visqueux, la mesure d'absorbance s'effectue à $600 \mathrm{~nm}$, directement sur tubes calibrés de $16 \mathrm{~mm}$ (Prolabo $320 \mathrm{R}$ )

Pour la correction de témoin "à blanc» on remplace le formol par de l'eau dans le réactif précédent.

L'apparition d'une teinte grisâtre (traces) ou d'une couleur bleu-violacé franche révèle la présence de glycoalcaloïdes. 
L'évaluation pondérale se fait par rapport à un étaIon alpha-solanine commercialisé (Sigma). On obtient la linéarité des mesures pour une gamme entre 0,1 et $0,8 \mathrm{mg}$ dans le prélèvement.

\section{Fractionnement et caractérisation des sapo- nosides stéroïdiques par chromatographie sur couche mince}

L'extrait isobutanolique est analysé par chromatographie sur couche mince sur un support usuel de gel de silice (G 1500 de Schleicher et Schüll) d'épaisseur $0,25 \mathrm{~mm}$ sur plaques $(20 \mathrm{~cm} \times 20 \mathrm{~cm})$. On peut aussi améliorer le fractionnement et la finesse des taches en utilisant la silice à hautes performances sur support aluminium (Kieselgel 60 F254-Merck-Ref. 5 548).

\section{Développement}

La séparation des hétérosides de la solasonine (GA) s'effectue généralement avec la phase supérieure du mélange : éluant $\mathrm{A}=$ Butanol primaire - Acide acétique - Eau (4-1-5; V/V). II doit être décanté plusieurs heures avant utilisation; son vieillisement (jusqu'à 10 j) peut améliorer la séparation des différentes taches de glycoalcaloïdes.

Le même éluant et les mêmes supports de CCM ont été retenus pour la séparation et l'identification des divers saponosides non azotés (SNA) du type furostanol (Sato \& Sakamura, 1973).

L'éluant $\mathrm{B}$ = chloroforme - méthanol - ammoniaque à $1 \%(2-2-1 ; V / V)$ est aussi utilisé pour séparer les hétérosides de la solasodine (Verbist, 1971).

\section{Révélation}

L'utilisation d'une gamme de réactifs relativement spécifiques permet de révéler les différentes fractions des saponosides stéroïdiques extraits (Wagner et al., 1984).

\section{Les saponosides stéroïdiques totaux}

Plus ou moins séparés, ils apparaissent en rouge-violacé, après pulvérisation d'une solution chloroformique saturée de trichlorure d'antimoine, et après chauffage quelques min de la plaque à $100-110^{\circ} \mathrm{C}$. Tous les noyaux stéroïdiques sont localisés sans distinguer GA et SNA.

\section{Les glycoalcaloïdes (GA)}

Ils sont révélés en taches de couleur orangé-brun sur fond blanc-jaunâtre, par le réactif classique de Dragendorff à l'iodobismuthate de potassium (Prolabo - code 30.989.236).

\section{Les saponosides non azotés (SNA) du type furostanol}

Ils donnent des taches de couleur rouge vif sur fond blanc-jaune avec le réactif d'Ehrlich $(2 \mathrm{~g}$ de diméthylaminobenzaldéhyde dans $100 \mathrm{ml}$ d'éthanol-chlorhydrique $2 \mathrm{~N}$ à $10 \%$ ) après chauffage de la plaque à 100 $110^{\circ} \mathrm{C}$. La spécificité de cette réaction pour la structure glycoside furostanol stéroïdique a été explicitée par Konishi et al. $(1984,1985)$.
Les saponosides non azotés (SNA) du type spirostanol

Ces saponosides aux noyaux $F$ non ouverts, ne sont pas révélés par le réactif précédent (Kintia \& Shvets, 1985a). Ces hétérocycles oxygénés sont visualisés par réaction avec la $p$-anisaldéhyde (Baccou et al., 1977, 1983; Kiyosawa \& Hutoh, 1968).

\section{Estimation de la teneur en saponosides par CCM}

Les étalons de référence sont: d'une part pour les divers alcaloïdes, l'alpha-solanine (Sigma), d'autre part pour les dérivés SNA du type furostanol, un des hétérosides séparés selon Sato \& Sakamura (1973). Dans cette dernière préparation, reprise par Cocallemen (1983), nous avons retenu une fraction chromatographique ( $F$ 155-160) correspondant au melongoside $P$ (ou à un hétéroside de structure et poids moléculaires très voisins).

Sur une même plaque en chromatographie unidimensionnelle, on juxtapose les extraits isobutanoliques avec un témoin (GA ou SNA) ou une gamme étalon de concentrations connues avec des dépôts de 5 à $20 \mu \mathrm{g}$.

Les confrontations variétales se font en déposant $50 \mu \mathrm{l}$ de chaque échantillon. Aprés révélation par un des réactifs spécifiques précédents, on effectue un classement visuel des taches séparées, sur 2 à 4 répétitions avec permutation sur les plaques. Cette estimation semi-quantitative est affinée en effectuant des dépôts variables (5 à $200 \mu \mathrm{l})$ selon la concentration de l'extrait. Généralement, on compare 3 extraits d'aubergines avec 3 dépôts de la gamme étalon correspondant à un type de saponoside. La teneur d'un même extrait est évaluée sur l'ensemble des taches révélées et sur plusieurs examens de différentes plaques.

\section{Evaluation de l'activité biologique des extraits}

Un contrôle des propriétés biochimiques membranaires des extraits totaux préparés de saponosides, sans distinction GA ou SNA, a été réalisé par deux test classiques d'utilisation usuelle.

\section{Activité hémolytique}

On a utilisé des érythrocytes (de sang humain), lavés et décantés dans du sérum physiologique tamponné à $\mathrm{pH} 7,4$. On compare en tubes à essais l'apparition d'une couleur rouge dans le surnageant. On détermine, par évaluation visuelle, le tube renfermant la concentration en saponoside juste suffisante pour obtenir l'hémolyse totale de la suspension à $2 \%$ de sang défibriné (Brunel, 1949).

\section{Indice de mousse}

Ce contrôle physique de la tensio-activité des saponosides s'effectue sur les extraits préalablement désalcoolisés. Ces estimations conventionnelles, selon le protocole de la Pharmacopée française (1965, 8e édit., p. 1 491), donnent l'importance et la stabilité relative des mousses formées par agitation en tubes à essais. 


\section{Appréciation de la qualité gustative des fruits}

L'analyse sensorielle, par quelques personnes du laboratoire, porte sur une évaluation de la saveur plus ou moins agréable də fractions de pulpe, fraîche ou après cuisson, dans une solution saline (Aubert, 1971).

\section{RÉSULTATS}

Les glycoalcaloïdes dérivés de la solasodine (GA) du fruit de l'aubergine sont essentiellement localisés dans la zone de pulpe placentaire du fruit comprenant les graines (Tableau I). La zone pédonculaire apparaît plus pauvre. Ce résultat est vérifié, quelle que soit la technique de dosage utilisée (colorimétrie ou chromatographie sur couche mince).

La variété traditionnelle «Violette de Barbentane» s'avère particulièrement riche en glycoalcaloïdes, mais sur des fruits cueillis à maturité excessive et inconsommables. Au cours d'une analyse sensorielle, le gel vert autour des graines est perçu comme ayant une forte sapidité amère et caustique.

Les saponosides non azotés (SNA) du type furostanol (Tableau II), généralement localisés dans les graines (Kintia \& Shvets, 1985 a et b), sont également présents dans le mésocarpe, en

Tableau I. Teneur et localisation des glycoalcaloïdes.

\begin{tabular}{|c|c|c|c|}
\hline \multicolumn{2}{|c|}{ Variétés } & $\begin{array}{l}\text { "Violette de } \\
\text { Barbentane" }\end{array}$ & "Dourga" \\
\hline \multicolumn{4}{|c|}{$\begin{array}{l}\text { Epicarpe (zone } \\
\text { épidermique) }\end{array}$} \\
\hline \multicolumn{2}{|c|}{ Colorimétrie (Clarke) } & $(0,5$ à 1$)$ & traces \\
\hline $\mathrm{CCM}$ & (Dragendorff) & traces & traces \\
\hline \multicolumn{4}{|c|}{$\begin{array}{l}\text { Mésocarpe sans graines } \\
\text { (pulpe du tiers inférieur } \\
\text { près du pédoncule) }\end{array}$} \\
\hline \multicolumn{2}{|c|}{ Colorimétrie } & $1,5(1$ à 2$)$ & $1(0,5$ à 1,5$)$ \\
\hline \multicolumn{2}{|c|}{ CCM } & $4 \quad$ (2 à 5$)$ & $2(1$ à 4$)$ \\
\hline \multicolumn{4}{|c|}{$\begin{array}{l}\text { Mésocarpe avec graines } \\
\text { (pulpe placentaire) }\end{array}$} \\
\hline Extrait à & Colorimétrie & 36 (31 à 39) & $7(5,5$ à 8$)$ \\
\hline froid & $\mathrm{CCM}$ & 30 (20 à 40) & $11(6$ à 13) \\
\hline Extrait à & Colorimétrie & 38 (33 à 43) & 9 (6 à 11) \\
\hline chaud & $\mathrm{CCM}$ & 30 (20 à 40) & 11 (8 à 16) \\
\hline
\end{tabular}

Conditions d'analyse et observations:

- Teneurs en mg de solanine étalon pour $100 \mathrm{~g}$ de matière fraîche.

- Fruits récoltés à un stade de maturité avancée (graines colorées).

- Moyennes (valeurs extrêmes) pour 4 déterminations dans différentes conditions d'évaluation d'un même extrait.

- En CCM, éluant $A$ ou $B$, toujours 2 taches pour des teneurs supérieures à $2 \mathrm{mg}$; chez ces deux variétés, une tache de $R f$ plus élevé $(0,36)$ est évaluée à 2 ou 3 fois celle de Rf́ bas $(0,32)$.
Tableau II. Teneur et localisation des saponosides SNA du type furostanol.

\begin{tabular}{|c|c|c|}
\hline Variétés & $\begin{array}{l}\text { "Ronde de } \\
\text { Valence" }\end{array}$ & "Dourga" \\
\hline $\begin{array}{l}\text { Mésocarpe sans graines } \\
\text { (pulpe du tiers inférieur } \\
\text { près du pédoncule) }\end{array}$ & $\begin{array}{c}1(0,5 \text { à } 2,5) \\
1 \text { tache }\end{array}$ & $\begin{array}{l}1(0,5 \text { à } 2,5) \\
1 \text { tache }\end{array}$ \\
\hline $\begin{array}{l}\text { Mésocarpe avec graines } \\
\text { (pulpe placentaire) }\end{array}$ & $\begin{array}{l}8(5 \text { à } 9) \\
3 \text { taches }\end{array}$ & $\begin{array}{c}2(0,5 \text { à } 3,5) \\
1 \text { taches }\end{array}$ \\
\hline $\begin{array}{l}\text { Même prélèvement } \\
\text { broyage très poussé }\end{array}$ & $\begin{array}{c}10(5 \text { à } 12,5) \\
3 \text { taches }\end{array}$ & $\begin{array}{l}3(2 \text { à } 4,5) \\
2 \text { taches }\end{array}$ \\
\hline
\end{tabular}

Conditions d'analyse et observations:

- Teneurs par CCM exprimés en mg pour $100 \mathrm{~g}$ de matière fraîche.

- Fruits récoltés à un stade de maturité avancée (graines colorées).

- Moyennes (valeurs extrêmes) pour 3 prélèvements de divers fruits et dans différentes conditions de révélation par le réactif d'Ehrlich.

- En CCM, éluant A, 3 taches pour "Ronde de Valence": Rf $0,40(15 \%), 0,34(75 \%)$ et $0,30(10 \%)$.

quantité non négligeable dans la pulpe dépourvue de graines apparentes.

Les variétés "Ronde de Valence» et «Dourga" ont des teneurs relativement importantes, mais dont l'extraction complète nécessite un broyage très poussé des graines.

Les teneurs en GA et SNA type furostanol diffèrent sensiblement suivant les variétés (Tableau III et IV). La sapidité apparaît d'autant plus forte que la teneur totale en saponosides est élevée.

Dans ces comparaisons variétales, "Dourga" a une sapidité relativement moins prononcée que les autres variétés, même à surmaturité, avec des graines très apparentes. Mais, la saveur douce n'est pas systématiquement associée à la couleur blanche du fruit (Tableau III) : si les variétés "Dourga" et "C8" sont de saveur douce, par contre, la variété «H5» est de saveur si forte qu'elle en est désagréable.

Par ailleurs, la richesse du fruit en saponosides stéroïdiques apparaît comme un caractère dominant dans les croisements (Tableau IV). Les hybrides « $F 1$ : Ronde de Valence $X$ Dourga" et «F1: Dourga X Ronde de Valence» ont des teneurs voisines de celles du parent le plus riche, "Ronde de Valence». Les hybrides réciproques ne diffèrent pas sensiblement entre eux, il n'y a pas d'effet cytoplasmique sur ce caractère. On note que les teneurs en saponines du type furostanol sont plus élevées en F1 que chez les parents. 
Tableau III. Relations entre saveur et teneur en saponosides. (Influence de la variété).

\begin{tabular}{|c|c|c|c|c|}
\hline Variétés & $\begin{array}{l}\text { "Ronde de } \\
\text { Valence" }\end{array}$ & «Dourga» & «C8» & $« H 5 »$ \\
\hline Poids moyen des fruits & $335 \mathrm{~g}$ & $212 \mathrm{~g}$ & $260 \mathrm{~g}$ & $155 \mathrm{~g}$ \\
\hline $\begin{array}{l}\text { Glycoalcaloïdes (GA) } \\
\text { (colorimétrie) }\end{array}$ & $5( \pm 2)$ & $1,5( \pm 1)$ & $1,5( \pm 1)$ & $11( \pm 3)$ \\
\hline Furostanol saponines (SNA) & $7( \pm 2)$ & $3,5( \pm 1)$ & $3,0( \pm 2)$ & $5( \pm 2)$ \\
\hline en CCM & 3 taches & 2 taches & 1 tache & 2 taches \\
\hline Total & 12 & 5 & 4,5 & 16 \\
\hline Sapidité avant et après cuisson & forte & douce & douce & désagréable \\
\hline
\end{tabular}

Conditions d'analyse et observations:

- Fruits récoltés à maturité commerciale (ou légèrement dépassée, surtout H5); Dourga, $\mathrm{C} 8$ et $\mathrm{H} 5$ ont des fruits blancs.

- Moyennes ( \pm valeurs extrêmes) pour 3 ou 4 déterminations sur un extrait de chaque lot.

Tableau IV. Contrôle de l'activité hémolytique et tensio-active des extraits de différents génotypes.

\begin{tabular}{|c|c|c|c|c|c|c|}
\hline $\begin{array}{l}\text { Variété ou } \\
\text { croisement }\end{array}$ & $\begin{array}{l}\text { "Ronde de } \\
\text { Valence" }\end{array}$ & "Dourga" & «C8» & $« H 5 »$ & $\begin{array}{c}F 1 \\
R \vee \times D O\end{array}$ & $\begin{array}{c}F 1 \\
D O \times R V\end{array}$ \\
\hline $\begin{array}{c}\text { Glycoalcaloïdes } \\
\text { (colorimétrie) }\end{array}$ & 3,7 & 1,5 & 1,0 & 7,2 & 3,8 & 3,7 \\
\hline $\begin{array}{l}\text { Furostanol saponines } \\
\text { (CCM) }\end{array}$ & 6,5 & 4,2 & 3,0 & 7,5 & 11,2 & 10,3 \\
\hline Total & 10,2 & 5,7 & 4,0 & 14,7 & 15 & 14 \\
\hline Sapidité & forte & douce & douce & désagréable & très forte & très forte \\
\hline $\begin{array}{l}\text { Indice de mousse } \\
\text { relatif }\end{array}$ & ++ & + & + & ++ & ++++ & +++ \\
\hline $\begin{array}{l}\text { Activité hémolytique } \\
\text { relative }\end{array}$ & ++ & + & + & +++ & $++t$ & +++ \\
\hline
\end{tabular}

Conditions d'analyse et observations:

- Teneurs en mg pour $100 \mathrm{~g}$ de matière fraîche (Etalons sur Tableaux I et II).

- Fruits récoltés à maturité commerciale (ou légèrement dépassée).

- F1 RV XDO = F1 «Ronde de Valence" $X$ «Dourga" et F1 DOX RV = F1 «Dourga" $X$ «Ronde de Valence".

- Moyennes pour 2 déterminations sur un seul extrait de chaque lot.

L'activité biologique des extraits, évaluée par le pouvoir hémolytique et l'indice de mousse (Tableau IV), apparaît liée à la teneur totale en saponosides stéroïdiques. Mais, nous ne pouvons préciser si ces propriétés sont plus par-ticulièrement le fait des GA ou des SNA type furostanol, ainsi que des hétérosides ou des aglycones.

Globalement, pour les fruits pris au stade commercial (Tableaux III et IV), nos évaluations pondérales en saponosides totaux se situent entre 4 et $16 \mathrm{mg}$ pour $100 \mathrm{~g}$ de matière fraîche. Les limites vont de 1 à $11 \mathrm{mg}$ pour les GA (dosés par rapport à l'étalon alpha-solanine), et de 3 à $11 \mathrm{mg}$ pour les SNA type furostanol. On notera que nos évaluations sont incomplètes, puisque nous n'avons pas mesuré les SNA type spirostanol.

\section{DISCUSSION}

\section{Réserves méthodologiques et caractérisa- tion des extraits}

Les opérations d'extraction des saponosides stéroïdiques, comportent des risques d'hydrolyse des hétérosides. Mais, la purification et le fractionnement sont nécessaires, compte-tenu du nombre limité de réactifs spécifiques des glycoalcaloïdes et des saponosides non azotés (Mahato et al., 1982; Price et al., 1987). 
La chromatographie sur couche mince révèle différentes taches correspondant à la présence dans les extraits d'un mélange complexe d'hétérosides chez les glycoalcaloïdes (tableau I) et chez les saponosides non azotés (tableau II). Ces dérivés distincts sont estimés à l'aide d'une codification semi-quantitative visuelle, dans un mode d'évaluation relativement imprécis. Dans les meilleures conditions d'analyse en CCM, l'erreur expérimentale serait de l'ordre de $20 \%$ pour différents extraits purifiés de saponines de diverses origines (Fenwick \& Oakenfull, 1983). Elle est fonction du nombre d'essais ( 3 à 4 répétitions) et surtout, du facteur de dilution des extraits par rapport aux étalons déposés. Elle peut être chiffrée, dans notre cas, entre 20 et $50 \%$, pour des teneurs comprises entre 20 et 2 $\mathrm{mg}$ pour $100 \mathrm{~g}$ de matière fraîche. Pour les faibles teneurs, l'évaluation devient aléatoire et de peu d'intérêt.

Néanmoins, cette méthode usuelle adoptée par des sélectionneurs sur la pomme de terre (Coxon et al., 1979; Coxon \& Geraint-Jones, 1981, voir référence Filadelphi et Zitmak, 1983) nous semble préférable à des indices colorimétriques donnant un dosage global et de spécificité incertaine.

Les indices d'activité biologique donnent des résultats imprécis et aléatoires. De nombreuses substances peuvent interférer dans la formation des mousses. De même, l'activité hémolytique, critère classique discuté par Jones \& Elliot (1969), a été utilisée par Juvik \& Stevens (1982) puis Juvik et al. (1982) pour le dosage de la tomatine (GA). Douillard \& Marion (1987) l'ont associé à la CCM comme révélateur; cette technique pourrait être reprise dans de nouvelles analyses.

Selon les techniques d'extraction et de dosage utilisées, les teneurs en glycoalcaloïdes d'un même matériel végétal peuvent fluctuer énormément. Par exemple, pour la pomme de terre (Clement \& Verbist, 1980) le facteur de variation est de 1 à 10. Néanmoins, les deux méthodes de dosage des glycoalcaloïdes que nous avons utilisées (colorimétrie et chromatographie sur couche mince), donnent des résultats voisins, compte tenu de la forte variabilité des mesures pour un même lot (Tableau I). Malgré cette imprécision, les variations de teneur entre zones du fruit et entre variétés sont nettes (Tableaux I à IV).

Les teneurs en glycoalcaloïdes que nous avons trouvées sont comparables à celles données dans les rares références bibliographiques disponibles sur l'aubergine, avec des réserves, car les méthodes de mesure sont différentes. Jones \& Fenwick (1981) trouvent une teneur de 8 $\mathrm{mg}$ (pour $100 \mathrm{~g}$ de matière fraîche) sur deux échantillons de fruits d'origine non précisée. Ils utilisent le dosage colorimétrique de Coxon et al. (1979), plutôt adapté à la mesure des fortes teneurs en solanine et chaconine de la pomme de terre. Bajaj et al. (1979) situent la teneur en glycoalcaloïdes des variétés indiennes d'aubergines entre 6 et $20 \mathrm{mg}$, exprimée en solanine, par une méthode colorimétrique globale discutable. Néanmoins, les écarts apparents entre nos résultats et ceux de ces auteurs, obtenus par des techniques très différentes, sont tout à fait admissibles. Ils montrent néanmoins la nécessité de l'adoption d'une norme universelle pour doser ces mélanges de composés.

L'identification exacte des différents hétérosides révélés par la CCM n'a pas été faite.

Quelques essais d'isolement de différents dérivés de la solasodine n'ont pas abouti, compte tenu des faibles teneurs et de l'instabilité des hétérosides. La préparation ou l'utilisation de dérivés connus (alpha-solasonine, bêta-solamargine ou autres) s'avère difficile, avec de nettes différences selon les sources végétales et les fournisseurs.

Ces imprécisions analytiques ne sont pas inadmissibles compte tenu de nos objectifs. La connaissance exacte des nombreux dérivés de la solasodine (leptines, solasonines alpha, bêta ou gamma, solamargine ou solamarine, soladiène de dégradation, etc.) et des différents saponosides non azotés (mélongosides du type furostanol $N, O$ et $P$ et du type spirostanol $L$ ou $M$ ) n'est pas indispensable, si l'on fait le postulat d'une activité biologique similaire chez toutes ces substances notamment sur l'amertume. Cette hypothèse probable reste à vérifier. De même, les structures spécifiques, responsables du pouvoir moussant ou de l'activité hémolytique, sont encore controversées (Oakenfull, 1981; Price 1987).

Dans les saponosides non azotés, les dérivés SNA cyclisés type spirostanol nous semblent être présents en très petites quantités pour des fruits récoltés au stade commercial. Pour les faibles concentrations, les artefacts méthodologiques sont problables (notamment au niveau de la révélation avec des chromophores jaunes peu spécifiques); c'est pourquoi nous ne les avons pas quantifiés. Nous nous sommes donc limités au dosage des SNA type furostanol, comme Kawano et al., (1975) et Sato \& Sakamura (1973) au cours de l'analyse des composants amers des turions d'asperge et des graines de tomate. On peut admettre que les structures du type furostanol seraient les précurseurs des formes du type spirostanol (Kiyosawa \& Hutoh, 1968). Les SNA type spirostanol n'apparaîtraient que secondairement dans la plante. Leur dosage colorimétrique n'est envisageable que pour les 
espèces végétales qui en contiennent relativement beaucoup (Baccou et al., 1977, 1983).

Ultérieurement, l'analyse individuelle des alcaloïdes et des saponosides non azotés pourrait être poursuivie, notamment avec des techniques comme la chromatographie liquide haute performance (CLHP), mais qui présentent l'inconvénient d'être longues et coûteuses. Nos analyses en chromatographie sur couche mince (CCM) en constituent une approche plus à la portée de très nombreux laboratoires de sélectionneurs.

\section{Sapidité des fruits, intérêt alimentaire et technologique}

La teneur des fruits en saponosides totaux (GA et SNA) est nettement reliée à la saveur (Tableaux III et IV). Le seuil d'agrément gustatif pourrait se situer entre 5 et $10 \mathrm{mg}$ de saponosides totaux pour $100 \mathrm{~g}$ de matière fraîche. La valeur inférieure $(5 \mathrm{mg})$ donne une perception agréable; la limite supérieure (10 mg) correspond à une sapidité excessive. Si l'on ne considère que les teneurs en glycoalcaloïdes, les limites d'acceptabilité sont abaissées à 3,7 mg (saveur forte) et 7,2 mg (sapidité désagréable). Les saponosides non azotés (SNA) apparaissent jouer un rôle complémentaire dans la saveur amère. Ces niveaux de la sensibilité gustative s'accordent avec les données bibliographiques relatives à la sapidité des alcaloïdes d'autres Solanacées (Aubert, 1971; Maga 1980; Zitmak \& Filadelphi, 1985).

Les effets des saponosides stéroïdiques sur la santé humaine restent très controversés, en particulier ceux des saponosides non azotés, souvent considérés comme moins toxiques que les alcaloïdes (Birk \& Peri, 1980; Fenwick \& Oakenfull, 1983; George, 1965; Gibney et al., 1982; Mahato et al., 1982; Oakenfull \& Topping 1983; Sharma et al., 1983). Le seuil maximum d'acceptabilité sanitaire généralement admis est d'environ $20 \mathrm{mg}$ en solanine pour $100 \mathrm{~g}$ de matière fraîche sur la pomme de terre. Mais le rejet gustatif des consommateurs intervient dès que les teneurs des aubergines en saponosides stéroïdiques totaux atteignent $15 \mathrm{mg}$ pour $100 \mathrm{~g}$ de matière fraîche (Tableaux IV et $V$ ). Ce risque sanitaire est donc réduit, d'autant plus que les pratiques culinaires, notamment la cuisson, réduisent la teneur en alcaloïdes (Kibler et al., 1985). on notera cependant qu'une saveur forte est quelquefois recherchée par certains consommateurs.

Sur le plan technologique, les saponosides peuvent aussi avoir un effet indirect sur la couleur, la texture et le goût des préparations culi- naires et industrielles d'aubergines (Aubert, 1981). Par exemple, la rétention d'huile par friture apparaît favorisée par ces composés tensioactifs (résultats non publiés).

Enfin, la formation de mousses intéresse aussi diverses préparations industrielles à base de divers fruits de Solanacées (jus, pulpes et concentrés de tomates; plats cuisinés). Des études ultérieures pourraient suivre l'influence de ces saponosides dans la qualité des denrées transformées par la cuisson.

\section{CONCLUSIONS}

Les techniques d'extraction et de dosage proposées, bien que partielles et discutables, confirment la richesse relative des fruits de l'aubergine en glycoalcaloïdes (GA) et en saponosides stéroïdiques non azotés (SNA) du type furostanol.

Ils sont essentiellement localisées dans la zone placentaire, mais on note leur présence non négligeable dans la pulpe sans graines apparentes.

La participation de ces composés à la saveur typique et à la qualité alimentaire de ce légumefruit est comparable à celle d'autres produits semblables trouvés dans les solanacées, pomme de terre et tomate notamment. Les seuils de perception et d'agrément, sont situés entre 5 et $10 \mathrm{mg}$ pour $100 \mathrm{~g}$ de matière fraîche, exprimés en alpha-solanine ou en saponosides stéroïdiques de structure chimique et de poids moléculaire très voisins. Nos mesures révèlent que de nombreux fruits dépassent les limites admissibles d'acceptabilité gustative.

Nous n'avons pas poussé l'analyse de ces constituants hétérosidiques au delà de cette subdivision glycoalcaloïdes et SNA du type furostanol. Des dérivés cyclisés type spirostanol, vraisemblablement moins abondants, du moins dans les fruits au stade commercial, sont volontairement négligés dans ces évaluations.

Nos observations montrent que les saponosides sont trouvés plus abondamment dans les fruits de récoltes tardives inconsommables. Des études ultérieures plus systématiques seront nécessaires pour préciser l'influence exacte sur la sapidité du stade physiologique de maturité à la cueillette.

Le rôle des facteurs génétiques sur la saveur des fruits est mis en relief dans cette expérimentation préliminaire. "Dourga» et «Ronde de Valence", variétés aux qualités organoleptiques très contrastées, ont des teneurs en saponosides stéroïdiques très différentes. Par ailleurs, nous avons montré que la couleur blanche de l'épider- 
me n'est pas toujours associée à une saveur douce. Enfin, la richesse en saponosides apparaît comme un caractère à hérédité dominante, puisque les hybrides entre "Dourga" et "Ronde de Valence" ont des teneurs voisines de celles de leur parent le plus riche, «Ronde de Valence».

Toutefois, sur diverses récoltes, l'influence des facteurs agro-climatiques et leur interaction avec les caractéristiques génétiques seront à considérer, préalablement par les voies usuelles proposées.

\section{RÉFÉRENCES}

Arveiller R. (1970) Les noms français de l'aubergine. Rev. Linguistique Romane, 225-244

Aubert S. (1971) L'aubergine (Solanum melongena L.) 1 - Composition et facteurs de qualité. Ann. Technol. Agric. 20, 241-264

Aubert S. (1981) Tomatine et saponines stéroïdiques participant à la qualité organoleptique des produits de la tomate. p. 171-178. Actes du congrès EUROCARPIA (Association européenne de recherche en sélection végétale) du groupe de travail «Tomate». 18-21 mai 1981 C.R. Agronomique de Montfavet-Avignon. Station d'Amélioration des plantes maraîchères, Domaine St Maurice, F 84140 Montfavet

Aubert S. Pochard E. (1981) Problèmes de conservation en frais de l'aubergine (Solanum melongena L.) 1 - Etude bibliographique. P.H.M. Rev. Hortic. 215, 33-38. II - Essais préliminaires de mise au froid. P.H.M. Rev. Hortic. 216, 35-40

Baccou J.C., Lambert F. \& Sauvaire Y. (1977) Spectrophotometric method for the determination of total steroïdal sapogenin. Analyst 102, 458-465

Baccou J.C., Sauvaire Y., Rival A., Trisonthi P. \& Jonard R. (1983) Mise en évidence de l'identité structurale des saponines et des sapogénines stéroïdiques du fenugrec (Trigonella feonum-graecum $\mathrm{L}$.) produites par des suspensions cellulaires cultivées in vitro et chez les plantes de plein champ. C.R. Acad. Sci. (Fr.), 297 (Série III), 449-452

Bajak K.L., Kaur G. \& Chadha M.L. (1979) Glycoalkaloid content and other chemical constituents of the fruits of some egg plants (Solanum melongena L.) varieties. J. Plant Foods 3, 163-168

Birk Y. \& Peri I. (1980) Saponines. Chap. 6, 161-181. In: Toxic Constituants of Plant Foodstuffs, (Liener I.E. Ed.) Academic Press Inc., New York and London

Brunel, (1949) Saponosides (saponines). In: Traité Pratique de Chimie Végétale Tome III (Section technique d'agriculture tropicale). Imprim. Georges Frère, Tourcoing, 263-278

Cheeke P.R. (1976) Nutritional and physiological properties of saponines. Nutr. Rep. Int. 13, 315-324

Claringbold W.B., Few J.D. \& Renwick J.H. (1982) Kinetics and retentions of solanidine in man. Xenobiotica 12, 293-302

Clément E. \& Verbist J.F. (1980) Dosage de la Solanine dans le tubercule de Solanum tuberosum $L$. : Etude comparative de neuf méthodes colorimétriques. Lebensm. Wiss. Technol. 13, 202-206
Cocallemen S., (1983) Contribution à l'étude de constituants de graines de solanacées maraîchères: extraction et isolement des graines d'aubergine (Solanum melongena $L$.) - Mémoire de maîtrise de chimie et biologie végétale appliquées - Université de Perpignan (F 66025$)$ et Station de Technologie I.N.R.A. à Monfavet (F 84 140)

Coxon D.T., Price K.R. \& Jones P.G. (1979) Simplified method for the determination of total glycoalcaloids in potatoes. J. Sci. Food Agric. 30, 1043-1049

Coxon D.T. \& Geraint-Jones P. (1981) A rapid screening method for the estimation of total glycoalkaloids in potatoes. J. Sci. Food Agric. 32, 366-370

Crabbe P.G. \& Fryer C. (1980) Rapid quantitative analysis of solasodine, solasodine glycosides and solasodiene by high-pressure liquid chromatography. J. Chromatogr. 187, 87-100

Crabbe P.G. \& Fryer C. (1982) Evaluation of chemical analysis for the determination of solasodine in Solanum lacianatum. J. Pharma. Sci. 71, 1356-1362

Dalvie R.R et Bowie W.C. (1984) Toxicology of solanin: an overview. Vet. Hum. Toxicol. 25, 13-15

De Waal H.L., Neethling L.P. \& Perold G.W. (1960) The bitter principle of Solanum melongena (Egg plant) fruits. J. S. Afr. Chem. Inst. XIII, 45-47

Douillard R. \& Marion D. (1987) Répartition des saponines dans les produits obtenus lors de la préparation des protéines de feuille de luzerne. Sci. Aliment. (Fr.) 7, 135-146

Fenwick D.E. \& Oakenfull D. (1983) Saponin content of food plants home prepared foods J. Sci. Food Agric. 34, 186-191

Filadelphi M.A. \& Zitmak A. (1983) A simple TLC stan dard for identification of potato glycoalkaloïds. Can. Inst. Food Sci. Technol. J. 16, 151-153

Freeland W.J., Calcott P.H. \& Anderson L.R. (1985) Tannins and saponins: interaction in herbivore diets. Biochem. Syst. Ecol. 13, 189-193

Gelder W.M.J. (1984) A new hydrolysis technique for steroïd glycoalkaloids with unstable aglycones from Solanum spp. J. Sci. Food Agric. 35, 487-494

George A.J. (1965) Legal status and toxicity of saponins. Food Cosmet. Toxicol. 3, 85-91

Gibney M.H., Pathirama C. \& Smith L. (1982) Saponins and fibre. Atherosclerosis 45, 365-367

Jones M. \& Elliot F.C. (1969) Two rapid assays for saponins in individual Alfalfa plants. Crop Sci. 9, 688691

Jones P.G. \& Fenwick G.R. (1981) The glycoalkaloïd content of some edible Solanaceous fruit and potato products. J. Sci. Food Agric. 32, 419-421

Juvik J.A. \& Stevens M.A. (1982) Inheritance of foliar $\alpha$-tomatine content in tomatoes. J. Am. Soc. Hortc. Sci. 107, 1061-1065

Juvik J.A., Stevens M.A. \& Rick C.M. (1982) Survey of the genus Lycopersicon for variability in $\alpha$-tomatine content. Hortscience 17, 764-766

Kawano K., Sakai K., Sato H. \& Sakamura S., (1975) A bitter principle of asparagus: isolation and structure of furostanol saponin in asparagus storage root. Agric. Biol. Chem. 39, 1199-2002

Kibler V.R. Lang H. et Ziegler W., (1985). Einfluss küchentechnischer Massnahmen auf den Solaningehalt grüner Tomatenfrüchte. Dtsch. Lebensm.Rundsch 81, 111-113 
Kintia P.K. \& Shvets S.A. (1985a) Melongoside $L$ and melongoside $M$, steroidal saponins from Solanum melongena seeds. Phytochemistry 24, 197-198

Kintia P.K. \& Shvets S.A. (1985b) Melongosides N, O and $P$ : steroidal saponins from seeds of Solanum melongena. Phytochemistry 24, 1567-1569

Kiyosawa S. \& Hutoh M. (1968) Detection of proto-type compounds of diosgenin and other spirostanol-glycosides. Chem. Pharm. Bull. (Tokyo) 16, 1162-1164

Konishi T., Hiyosana S. \& Shoji J., (1984) Studies on the coloration mechanism of furostanol derivatives with Ehrlich reagent. I. On the reaction of 3,26-Dimethoxyfurost-5,20-diene with Ehrlich reagent. Chem. Pharm. Bull (Tokyo) 32, 2111-2116

Konishi T., Hiyosana S. \& Shoji J., (1985) Studies on the coloration mechanism of furostanol derivatives with Ehrlich reagent. II. On the reaction of furostanol glycoside with Ehrlich reagent. Chem. Pharm. Bull (Tokyo) 33, 591-597

Kritchevsky D., Tepper S.A. \& Story J.A. (1975) Influence of egg plant (Solanum melongena L.) preparation on chlolesterol metabolism in rats. Exp. Pathol. 10, 180-183

Maga J.A. (1980) Potato glycoalkaloids. C.R.C. Crit. Rev. Food Sci. Nutr. 12, 371-405

Mahato S.B., Ganguly A.N. \& Saav N.P. (1982) Steroid saponins (review). Phytochem.21, 959-978

Massiot G., Lavaud C., Guillaume D., Lemen-Olivier L. \& Van G., (1986) Identification and sequencing of sugars in saponins using 2D 1 H N.M.R. spectroscopy. J. Chem. Sci. 728, 1485-1487

Mitschek G.H.A., (1970) L'action de Solanum melongena en pathologie expérimentale. $Q$. J. Crude Drug. Res. 10, 1550-1555

Morgan B., Heald M., Brooks S.G. Tee J.L. \& Green J. (1972) The interactions between dietary saponins, cholesterol and related sterols in the chick. Poult. Sci. $51,677-682$

Oakenfull D.G. (1981) Saponins in food - a review. Food Chem. 6, 19-40

Oakenfull D.G. \& Sidhu G.S. (1983) A physico-chemical explanation for the effects of dietary saponins on cholesterol and bile salt metabolism. Nutr. Rep. Int. 27, 1253-1259

Oakenfull D.G. \& Topping D.L. (1983) Saponins and plasma cholesterol. Artherosclerosis 48, 301-303

Price K.R, Mellon F.A., Self R., Fenwick G.R. \& Osman S.F. (1985) Fast atom bombardement mass spectroscopy of solanum glycoalcaloids and its potential for mixture analysis. Biomed. Mass Spectro. 12, 79-85

Price K.R. Johnson I.T. \& Fenwick G.R (1987) The chemistry and biological significance of saponins in foods and feedingstuffs. C.R.C. Crit. Rev. Food Sci. Nutr. 26, 27-135

Regerat F. \& Pourrat H. (1981) Isolement par voie fermentaire de la solasonine à partir d'un mélange de glucoalcaloïdes. Planta Medica 43, 280-284

Sato H. \& Sakamura S. (1973) A bitter principle of tomato seed. Isolation and structure of a new furostanol saponin. Agric. Biol. Chem. 37, 225-231

Schreiber K. (1968) Steroid alkaloids: The solanum group. In: The alkaloïds - Chemistry and Physiology. (Manske R.M.F. Ed.) Academic Press Inc. , New York and London, 1-178

Sharma J.C., Chand R., Sati O.P. \& Sharma A.K. (1983) Oligofurostanosides from Solanum nigrum. Phytochemistry 22 (5), 1241-1244

Sidhu G.S. \& Oakenfull (1986) A mechanism for the hypocholesterolaemic activity of saponins. Bristish J. Nutr. 55, 643-649

Trione S.O. \& Cony M.A. (1988) Solanum elaeagnifolium cav., fuente de precursores esteroïdales. Analysis y perspectivas para su cultivo. Interciencia 13, 303337

Verbist J.F. (1971) Contribution à l'étude des glycoalcaloïdes des solanacées indigènes. Thèse de doctorat d'Etat en Pharmacie. U.E.R. Université de Nantes, France

Verbist J.F. \& Monnet R (1979) A propos de la teneur en solanine des petits tubercules nouveaux de pomme de terre (Solanum tuberosum L.) Potato Res. 22, 239244

Verbist J.F., Monnet R. \& Dobremez J.F. (1977) Les alcaloïdes stéroïdiques de sept solanum népalais: identification et teneur. Plant. Méd. Phytothér. 11, 4048

Vohora S.B., Kumar I. et Khan M.S.Y. (1984) Effect of alkaloïds of Solanum melongena on the central nervous system. J. Ethnopharmacol. 11, 331-336

Wagner H., Bladt S. \& Zgainski E.M. (1984) In: Plant Drug Analysis, Chap. Saponin Drugs 225-244. Springer Verlag, Berlin, New York

Weiler E.W., Kruger H. \& Zenk M.H. (1980) Radioimmunoassay for the determination of the steroidal alkaloid solasodine and related compounds in living plant and herbarium specimens. Planta medica 39, 112-124

Wu M.T. \& Salunkhe D.K. (1976) Devices for improving color development in glycoalkaloid determination. $J$. Food Sci. 4, 220-221

Zitmak A. \& Filadelphi M.A. (1985) Estimation of taste thresholds of three potato glycoalkaloids. Can. Inst. Food Sci. Technol. J. 48, 337-339 